\title{
Documentos digitalizados e elaboração de conhecimentos para a história da educação matemática
}

Digitized documents and knowledge development for the history of mathematics education

Victor Hugo de Lemos Gomes ${ }^{1}$

Wagner Rodrigues Valente ${ }^{2}$

\section{Resumo}

A crescente utilização de repositórios digitais, bem como a expansão do volume de documentos disponíveis para análise e uso como fontes de pesquisas, exigem uma adequação no modo de realizar investigações de forma a potencializar os seus resultados. O objetivo deste artigo é apresentar uma nova ferramenta para elaboração de conhecimento, no âmbito da história da educação matemática, apresentando-a por meio de um estudo de caso. Utilizando uma análise lexicométrica, aliada ao uso de softwares de processamento de dados, como o IRAMUTEQ, as pesquisas ganham a possibilidade de uso de múltiplos textos potencializando o processo de análise e diminuindo sobremaneira o tempo gasto no trabalho. Esse expediente permite o cruzamento de dados e informações tendendo a amplificar os resultados dos estudos em história da educação matemática.

Palavras-chave: história da educação matemática; IRAMUTEQ; Lexicometria; cruzamento de dados.

\section{Abstract}

The growing use of digital repositories, as well as the expansion of the volume of documents available for analysis and use as research sources, require an adaptation in the way of carrying out investigations in order to enhance their results. The aim of this article is to present a new tool for knowledge development, in the context of the history of mathematics education, presenting it through a case study. Using a lexicometric analysis, combined with the use of data processing software such as IRAMUTEQ, research gains the possibility of using multiple texts, enhancing the analysis process and greatly reducing the time spent at work. This expedient allows the crossing of data and information tending to amplify the results of studies in the history of mathematics education.

Keywords: history of mathematics education; IRAMUTEQ; Lexicometry; data crossing.

\footnotetext{
${ }^{1}$ Universidade Federal de São Paulo | victordelemos@gmail.com

${ }^{2}$ Universidade Federal de São Paulo | wagner.valente@unifesp.br
} 


\section{Introdução}

O uso de repositórios de conteúdos digitais tem sido prática constante nas pesquisas. Como lugar de guarda de arquivos, os repositórios armazenam, pelo menos, dois tipos deles: aqueles que se referem a textos e trabalhos já elaborados e publicados ou em processo de finalização e, também, os arquivos que contêm documentação que encerra dados a serem utilizados como fontes de pesquisa.

No âmbito das pesquisas relativas à história da educação matemática, seara que muito vem crescendo nos últimos anos, inserida no campo da Educação Matemática, também os repositórios vêm sendo utilizados. Há exemplos de muitos estudos compartilhados e projetos coletivos que se servem de repositórios como uma verdadeira base de dados. Neles há uma vasta documentação que reúne livros didáticos antigos, legislação escolar, revistas pedagógicas, cadernos de alunos dentre vários outros tipos de materiais para a pesquisa sobre o ensino de matemática e formação de professores ocorridos em outros tempos.

Como se disse, os estudos históricos sobre educação matemática têm crescido enormemente, trazendo contribuições aos estudos atuais de variadas formas. Seja, por exemplo, decifrando historicamente como foram sendo construídos currículos e programas de matemática; seja explicitando como e quando determinados conteúdos começaram a fazer parte dos ensinos; ou mesmo, foram eliminados das grades curriculares; estudos sobre mudanças ao longo do tempo de métodos, de didáticas, dentre tantos outros elementos que a perspectiva histórica traz como contribuições aos dias atuais do ensino de matemática.

A possibilidade de utilização de uma quantidade enorme de documentos digitalizados e disponíveis com acesso aberto pela internet vem relativizando um problema que era recorrente até tempos atrás: a dificuldade de obtenção de fontes de pesquisa, o moroso processo de encontrá-las, o impedimento de uso de documentos localizados geograficamente distantes dos pesquisadores, dentre outros entraves. Com os repositórios compartilhados, tomando como exemplo os estudos de história da educação matemática, tem-se rapidamente acesso a livros didáticos elaborados em diferentes estados brasileiros, revistas pedagógicas editadas em variados locais do país, cadernos de alunos e professores que cobrem quase todo o Brasil, para citar alguns exemplos. Assim, em tempos atuais, minimizou-se muito a dificuldade de encontrar materiais antigos para estudos históricos sobre o ensino de matemática. Eles, agora, encontram-se em boa medida digitalizados e alocados em repositórios ${ }^{3}$.

De outra parte, se o uso dos repositórios, dos materiais digitalizados, trouxe facilidades para o encontro das fontes de pesquisa, instaurou novos problemas para as investigações. Se em tempo anterior os estudos lançavam mão de uma quantidade limitada de documentos e materiais, resultante de um processo de busca individual de cada pesquisador junto a arquivos e centros de documentação, com os repositórios, uma enorme massa documental está disponível para uso nos estudos. Se antes o problema referia-se à

\footnotetext{
${ }^{3}$ Cite-se, como exemplo, a base de dados do GHEMAT Brasil - Grupo Associado de Estudos e Pesquisas em História da educação matemática, situado no endereço: https://repositorio.ufsc.br/handle/123456789/1769
} 
escassez de documentos à mão; agora, o desafio envolve o tratamento de uma gama enorme de materiais digitalizados.

As reflexões presentes neste artigo inserem-se nessa situação nova de pesquisa. Em específico, localiza-se no âmbito de estudos de história da educação matemática, tendo em vista o uso de repositório de conteúdo digital. Em específico, o texto retrata os processos que envolvem o desenvolvimento de projeto de pesquisa considerando a possibilidade de acesso a um grande número de artigos publicados em revistas pedagógicas de outros tempos, cujo assunto tratado refere-se ao ensino de matemática, em particular, ao ensino da aritmética nos primeiros anos escolares. O corpus documental utilizado refere-se a textos de orientação pedagógica aos professores sobre o trato dos conteúdos aritméticos.

Como utilizar um grande número de documentos digitalizados - como os artigos sobre o ensino de aritmética nos primeiros anos escolares - em prol do desenvolvimento de pesquisa sobre história da educação matemática? Esta questão norteia o desenvolvimento deste estudo.

\section{Uma referência para a pesquisa com documentos digitalizados}

Desde logo cabe dizer que a mobilização de grande número de documentos digitalizados requer a utilização de ferramentas de informática para tratamento desses documentos. Há possibilidades variadas de escolha desse instrumental.

Isso posto, este artigo tem como proposta apresentar o uso do software IRAMUTEQ como alternativa para trabalhos que demandem análises massivas de textos. De outra parte, toma como referência um exemplo de estudo uma pesquisa que utilizou revistas pedagógicas paulistas que veicularam orientações para o ensino de aritmética nos primeiros anos escolares, no período 1890-1920. Tal intervalo temporal é tido em estudos de História da Educação como época de vigência da chamada vaga pedagógica intuitiva.

A base para a realização da pesquisa foi composta por 33 artigos de aritmética publicados nas revistas pedagógicas paulistas no período mencionado. De outra parte, o processo de trabalho envolvido poderia reunir, em tese, um número ilimitado de documentos, não apenas três dezenas, como este estudo traz.

A análise comparativa de dois textos homogêneos, ou seja, com assuntos relacionados, tende a passar, entre outras coisas, pelo processo de leitura, entendimento da estrutura textual e linguística, distinção dos pontos de interesse que levaram a esses textos, sistematização das ideias que compõem a narrativa de cada um deles e inferências do pesquisador com o cruzamento das informações entre os documentos.

Por mais atento e detalhista que seja o pesquisador, quantos pontos o olho humano deixaria passar despercebidos ao realizar a análise de centenas de textos? E quanto tempo um único pesquisador demoraria para realizar esse trabalho? O enfrentamento dessas questões de modo ágil e sistematizado justifica, desde logo, a alternativa tratada no presente artigo.

A tecnologia dos softwares de processamento de dados aliada à lexicometria trazem a proposta de automatizar a análise de múltiplos textos e, com isso, reduzir substancialmente o tempo de análise. Lexicometria é por definição o tratamento estatístico de textos de forma a estruturar os conteúdos por meio dos vocabulários. Em outras palavras: 
A lexicometria é um procedimento metodológico e tecnológico - objetivo, descritivo, indutivo e científico - para tratar estatisticamente dados qualitativos sob fundo quantitativo para a caracterização topológica e combinatória de elementos lexicais de um corpus dado e delimitado, a fim de que a trajetória do discurso, por operações conhecidas e controladas, seja balizada, e a topologia e a combinatória dos elementos lexicais do corpus caracterizadas. (DAMASCENO, 2006, p.1116).

Segundo Damasceno (2006), a lexicometria permite identificar "co-relacionamentos e dados implícitos nos registros de um corpus, pelo estudo e desenvolvimento de um processo de extração", por meio da: identificação de dependências entre os vocabulários; identificação de desvios, no qual permite-se averiguar os elementos que se encontram fora dos padrões estabelecidos ou esperados; detecção e análise de estruturas e de vocabulários com características comuns e revelação de modelos capazes de descrever os vocabulários e conceitos envolvidos.

Todavia, uma metodologia deve ser desenvolvida ou utilizada de forma a permitir que o processo de lexicometria possa ser explorado. Damasceno (2006), indica que tais etapas dessa metodologia devem contemplar: pré-processamento - no qual os corpus passam por um processo de análise, reorganização e limpeza de dados; processamento: constituído pelo agrupamento, classificação, associações e visualização; e pós-processamento: inferências por meio da filtragem, seleção, ordenação, mapeamento e relação com fundamentos teóricos.

A chamada análise de conteúdo é uma metodologia que pode atender a tais demandas, seguindo-se os preceitos definidos por Bardin (2011), em seu livro "Análise de Conteúdo". Além das definições das etapas a serem seguidas, os estudos desse autor auxiliam no processo de análise, ou seja, como entender a lexicometria. A análise de conteúdo é por si só uma técnica de análise que "tende a valorizar o material a ser analisado, especialmente se a interpretação do conteúdo [...] estipular, como parâmetros, os contextos individuais, sociais e históricos nos quais foram introduzidos" (FRANCO, 2008, p.17). Em outras palavras, ao efetuar a análise de conteúdo é importante entender o contexto no qual o corpus está inserido.

Bardin (2011) estrutura a análise de conteúdo em cinco etapas: $1^{a}$ etapa - pré-análise, que se caracteriza pela separação do material de interesse e avaliação da representatividade estatística do material permitindo identificar a possibilidade ou não do prosseguimento da pesquisa; $2^{a}$ etapa - codificação, identificação de elementos que possam ser úteis para a análise, de forma a caracterizar um texto; $3^{a}$ etapa - categorização, criam-se categorias como forma de separar e classificar os textos; $4^{a}$ etapa - tratamento dos resultados, pode ocorrer com o auxílio de software de tratamento textual, submetendo os textos ao processo de lexicometria; e $5^{a}$ etapa - inferências e interpretação dos resultados.

Inúmeros softwares podem ser utilizados na $4^{a}$ etapa, como é o caso do IRAMUTEQ, que é uma ferramenta computacional gratuita e de código aberto, desenvolvida por Pierre Ratinaud. Tal software permite fazer análises estatísticas sobre corpus textuais, como: estatísticas textuais clássicas; Classificação Hierárquica Descendente (CHD) pelo método de Reinert; Análise de similitude; e Nuvem de palavras.

Isso posto, escolhemos realizar a análise lexicométrica de documentação de pesquisa no âmbito da história da educação matemática tendo por apoio o software IRAMUTEQ 
(http://www.iramuteq.org/) em conjunto com a biblioteca do software estatístico $R$ (https://www.r-project.org/).

Num primeiro momento, nota-se que a pesquisa com documentação histórica apresenta dificuldades específicas do ponto de vista de trabalho com materiais digitalizados. Quando o desenvolvimento da pesquisa depende de materiais históricos, com qualidade da imagem muitas vezes comprometida, a dificuldade aumenta, pelo fato de os softwares de análise textual dependerem dos textos, em formato txt, para serem manipulados.

Este foi o cenário da pesquisa em questão, no qual os materiais utilizados com fontes são antigos e encontram-se em imagens fixadas em arquivos PDF não pesquisáveis. Esse fato constitui um impeditivo para o uso da ferramenta IRAMUTEQ, que necessita dos textos para a serem pesquisáveis, manipuláveis. Para transformar as imagens de um PDF não pesquisável em pesquisável é necessário utilizar uma outra tecnologia computacional - o OCR (Optical Character Recognition) - que usa técnica para reconhecimento de caracteres em imagens e está presente em diversos softwares. Entretanto, quando falamos de documentos com qualidade comprometida, pois foram obtidos de originais já envelhecidos e com desgastes, tem-se outro desafio. Caberá tratar os documentos com técnicas de processamento digital de imagens. Aqui não caberia especificar ainda mais essa etapa, por distanciar-se dos objetivos deste texto. De todo modo, no estudo original que deu origem às reflexões que aqui estão sendo apresentadas, há um detalhamento do procedimento utilizado ${ }^{4}$.

\section{Fontes digitalizadas e exemplo de pesquisa em história da educação matemática}

O período selecionado para estudo, como se disse, é caracterizado como o da vigência do chamado método intuitivo para as práticas pedagógicas. Tem-se a presença marcante dos discursos educacionais baseados, sobretudo, nas ideias de Pestalozzi. Há, por esse tempo, uma nova era de pensar a educação: o ensino deveria apartar-se da tradição do aprender de cor, das práticas verbalísticas. Está em voga a ideia central de que os alunos aprendem por meio de coisas, do que hoje denominamos "materiais concretos". Por meio da intuição sensível sobre tais materiais, pensava-se, seria possível a aprendizagem do sistema numérico, das operações aritméticas, dentre outros conteúdos da aritmética escolar. Essa nova vaga pedagógica tem por tarefa levar o novo ideário do ensino para os professores, buscando as mudanças em suas práticas. Nesse sentido, as revistas pedagógicas jogam papel importante na divulgação das novas propostas.

As revistas pedagógicas editadas em São Paulo constituem vetores de divulgação do novo tempo pedagógico, ancoradas no pioneirismo paulista de criação dos grupos escolares, novo modelo de organização escolar, concebido na última década do século XIX.

De modo geral, as revistas buscam fazer circular modelos de lições e materiais que pretendem modular, dosar e aprimorar as práticas docentes de forma a tornar homogêneo o ensino (CARVALHO, 2000, p. 113).

\footnotetext{
${ }^{4}$ Trata-se da dissertação de mestrado de Gomes (2020).
} 
Consideramos que os resultados de estudos vindos da História da Educação, como os de Carvalho (2000) carecem aprofundamento em termos dos ensinos e propostas para a aritmética dos primeiros anos escolares.

Assim, ao analisar os conteúdos dos artigos das revistas pedagógicas paulistas elencados para a pesquisa, procura-se identificar e discutir quais as orientações circulam nos periódicos para o ensino de aritmética, correlacionando os resultados com pesquisas já desenvolvidas no campo da História da Educação.

A trajetória de realização das análises dos artigos das revistas paulistas considera, inicialmente, o repositório de conteúdo digital do GHEMAT Brasil, no qual foram coletados 33 artigos de aritmética publicados no período de 1890 a 1920. Os artigos de aritmética foram escolhidos com base nos programas curriculares disponibilizados nas revistas do período e nas regras de homogeneidade e pertinência, definidas por Bardin (2011).

Quadro 1- Relação de artigos analisados

BARRETO, A. Aritmética. Revista A Eschola Publica, 1896, Ano I, n. 4, dez., SP.

BARRETO, A. Aritmética. Revista A Eschola Publica, 1897, Ano II, n. 8, dez., SP.

BARRETO, A. Aritmética. Revista A Eschola Publica ano. 2, n. 5, mar., 1897, SP.

BARRETO, A. Aritmética. Revista A Eschola Publica, 1896, Ano I, n. 3, set., SP.

BARRETO, A. A Propósito da aritmética dos principiantes. Revista de Ensino, 1903, Ano I, n. 06, fev., SP.

BARRETO, A. A propósito da aritmética dos Principiantes. Apuros do Senhor Buarque! Rev. de Ensino, 1903, Ano I, n. 01, abr., SP.

BARRETO, A. O ensino da aritmética. Revista de Ensino, 1903 Ano I nr. 03 - agosto, SP.

BRITO, J. Cartas de Parker - Aritmética. Revista de Ensino, 1903, Ano I, n. 06, fev., SP.

CARDOSO, L. A Aritmética nas escolas. Revista do Ensino, 1912 Ano XI nr. 1 março.

C. A. R. Um pouco de Arithmética. Revista do Ensino, 1906 Ano V nr. 2 setembro.

DOLORES, D. A. Brinquedo didatico para ensinar a tabuada de multiplicação. Revista do Ensino, 1912 Ano XI nr. 1 março.

FAGUNDES, J. G. Distribuição das caixas. Revista de Ensino, nr. 3, setembro-dezembro, 1912, SP.

LEITE. F. E. A. Aritmética. Revista do Ensino, 1912 Ano XI nr. 1 março.

MACARIO, R. Diversão Aritmética. Revista de Ensino, 1904 Ano II nr. 06 - fevereiro, SP.

MONTEIRO, J. C. Diversão aritmética. Propriedades dos números invertidos. Revista de Ensino, 1904 Ano III nr. 03 - agosto, SP MONTEIRO. Aritmética Preliminar.. Revista de ensino nº 3 de 1916.

PENTEADO, F. Sua origem. Seus progressos. Seu ensino. Revista da Escola Normal de São Carlos, 1916, SP.

REVISTA DA ESCOLA NORMAL DE SÃO CARLOS. Média de números. Rev. Esc. Normal de S.Carlos, dez. 1919 ano IV, n 7

REVISTA DE ENSINO. Pedagogia prática Aritmética. Revista de Ensino, 1905 Ano IV nr. 03 agosto, SP.

REVISTA DE ENSINO. Pedagogia prática. Revista de Ensino, 1905 Ano IV nr. 01 abril, SP.

REVISTA DE ENSINO. Aula de contagem com pauzinhos coloridos. Revista de Ensino, 1912 Ano XI nr. 3 setembro-dezembro, SP.

THOMPSON, O. Aritmética Elementar. Revista A Eschola Publica, Agosto, 1893.

THOMPSON, O. Tabuada. Revista A Eschola Publica, Agosto, 1893.

THOMPSON, O. Tabuada. Revista A Eschola Publica, Setembro, 1893.

THOMPSON, O. Aritmética Elementar. Revista A Eschola Publica, Outubro, 1893.

THOMPSON, O. Aritmética Elementar. Revista A Eschola Publica, Janeiro, 1894.

THOMPSON, O. Aritmética Elementar. Revista A Eschola Publica, Março, 1894.

THOMPSON, O. Aritmética Elementar. Revista A Eschola Publica, Abril, 1894.

THOMPSON, O. Aritmética Elementar. Revista A Eschola Publica, Maio, 1894.

THOMPSON, O. Aritmética Elementar. Revista A Eschola Publica, Junho, 1894.

Fonte: Elaborado pelos autores

As regras de homogeneidade e pertinência definidas por Bardin (2011) avaliam se a quantidade de material é representativa para a pesquisa. Desta forma, entende-se que as fontes de pesquisa são homogêneas seguindo três critérios: as fontes são todas Revistas Pedagógicas; os materiais são de uma mesma cidade, São Paulo; o marco histórico de 1890 a 1920 tem definido um sistema educacional com organização semelhante, ou seja, mantendo o ensino com duração de quatro anos. A Regra de Pertinência verifica a 
adequação dos documentos como fonte de pesquisa de modo a servirem como hipótese de pesquisa. As Revistas Pedagógicas são consideradas como instrumentos com o objetivo de formar os professores mantendo uma padronização do ensino, fato que qualifica os textos desses periódicos na Regra de Pertinência. O Quadro 1, abaixo, mostra a relação dos artigos de revistas utilizados na pesquisa:

Após tratamento e organização, os textos foram processados pelo IRAMUTEQ, seguindo a seguinte ordem de análise:

- Análise da Nuvem de Palavras - que permite a identificação das palavras com maior número de ocorrências;

- Análise de Similitude - que utiliza a teoria dos grafos e permite identificar a conexão entre as palavras no texto;

- Análise da Classificação Hierárquica Descendente (CHD) - define-se um limiar de importância e faz-se a análise dos segmentos de textos de cada classe de forma a encontrar significado para as classes.

- Análise Fatorial de Correspondência - A partir da CHD, permite identificar as palavras de cada classe em quatro quadrantes, facilitando o entendimento de proximidade entre classes e palavras.

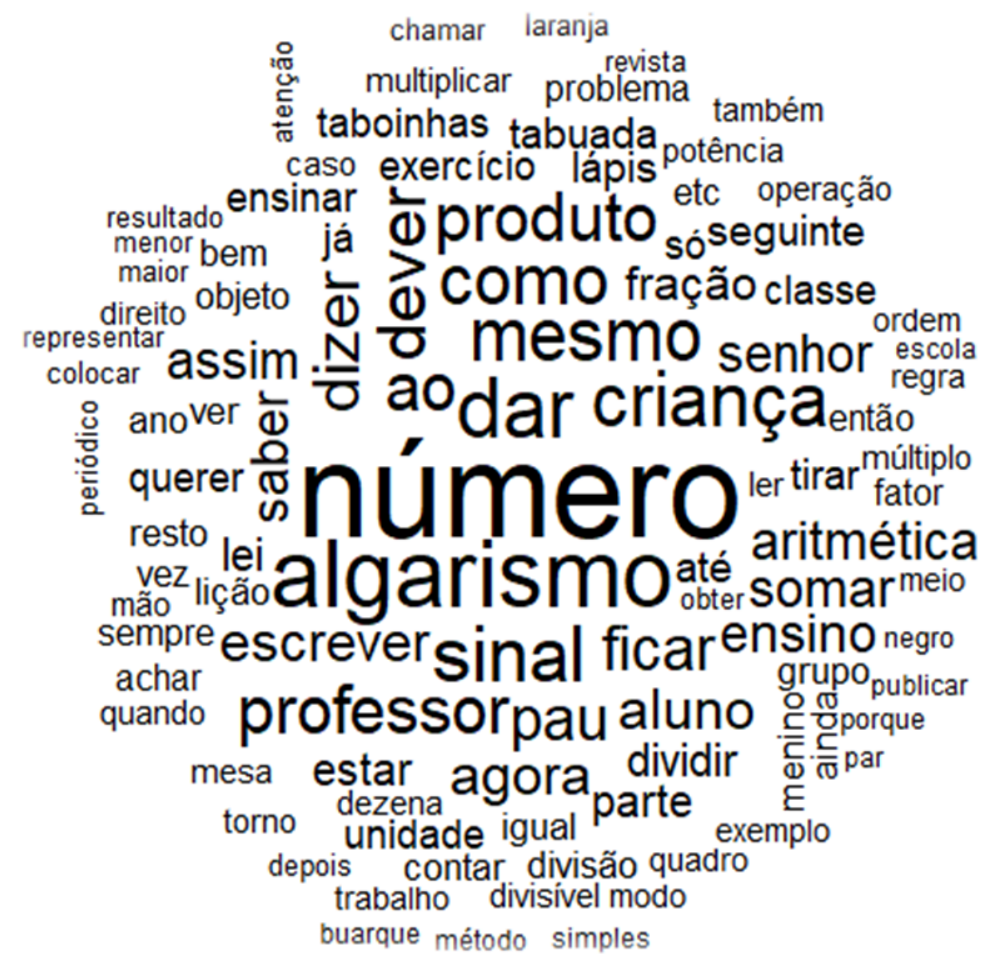

Figura 1 - Nuvem de Palavras dos 33 documentos de aritmética gerado pelo IRAMUTEQ. Fonte: IRAMUTEQ, 2019.

A nuvem de palavras permite uma visualização imediata e quantitativa das palavras que mais aparecem nos artigos de aritmética. De forma complementar, a Análise de Similitude permite o entendimento da ligação entre essas palavras, representado por meio de nós. Usando como exemplo (setas vermelhas), percebemos uma ligação indireta entre professor e criança, e chamamos de indireta pois a ligação passa pela palavra lápis, talvez indicando 
tentativas do professor de orientar a criança na execução de alguma tarefa com o lápis. Podemos ver essa mesma relação indireta entre professor e aluno. Essas ligações podem indicar uma tentativa dos autores dos artigos em mostrar aos professores como a interação para ensinar a criança/aluno deve ocorrer.

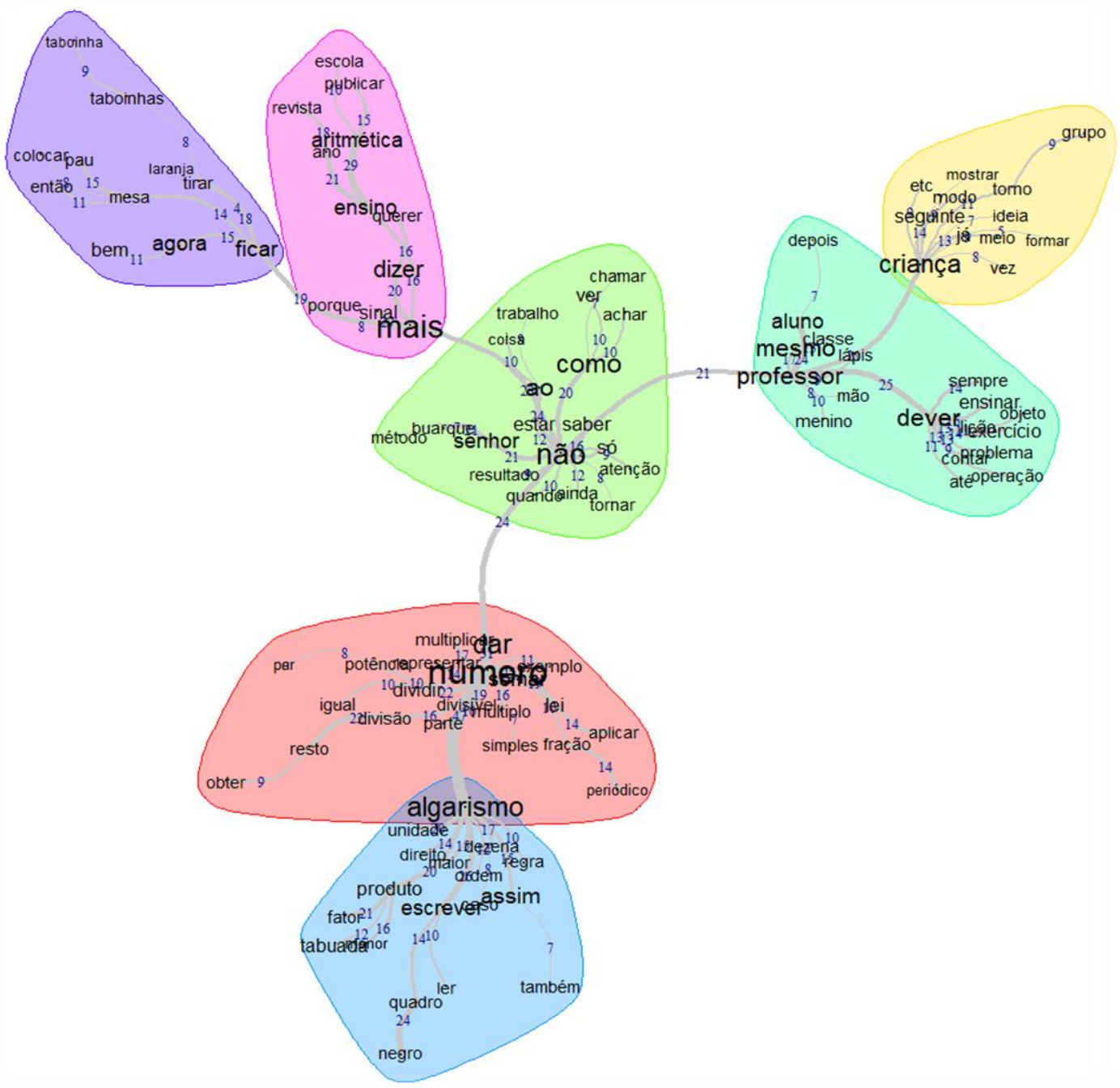

Figura 2 - Análise de Similitude dos 33 documentos de aritmética gerado pelo IRAMUTEQ. Fonte: IRAMUTEQ, 2019

A análise de similitude permite uma visão macro dos artigos de aritmética. Entretanto, não é possível fazer-se a classificação das palavras para entender com maior aprofundamento como os autores das revistas procuravam formar os professores, missão que fica a cargo das inferências do pesquisador sobre a Classificação Hierárquica Descendente (CHD).

As inferências do pesquisador sobre a CHD, revelam o que podemos denominar de uma anatomia das aulas, no âmbito da qual os autores das revistas pedagógicas paulistas procuravam formar os professores. Suas ações expressavam-se por meio de:

1. Métodos de ensino de aritmética (classe 4), por exemplo: Cartas de Parker;

2. Ações a serem seguidas pelo professor (classe 3), por exemplo: escrever no quadro negro; 
3. Etapas de ensino que revelavam os momentos para apresentar um objeto, um problema ou expor um conteúdo (classe 2);

4. Exemplos e exercícios para uso do professor e aluno (classe 6).

Em outras palavras, os autores das revistas procuraram criar um modelo de aula que pudesse ser seguido pelos professores.

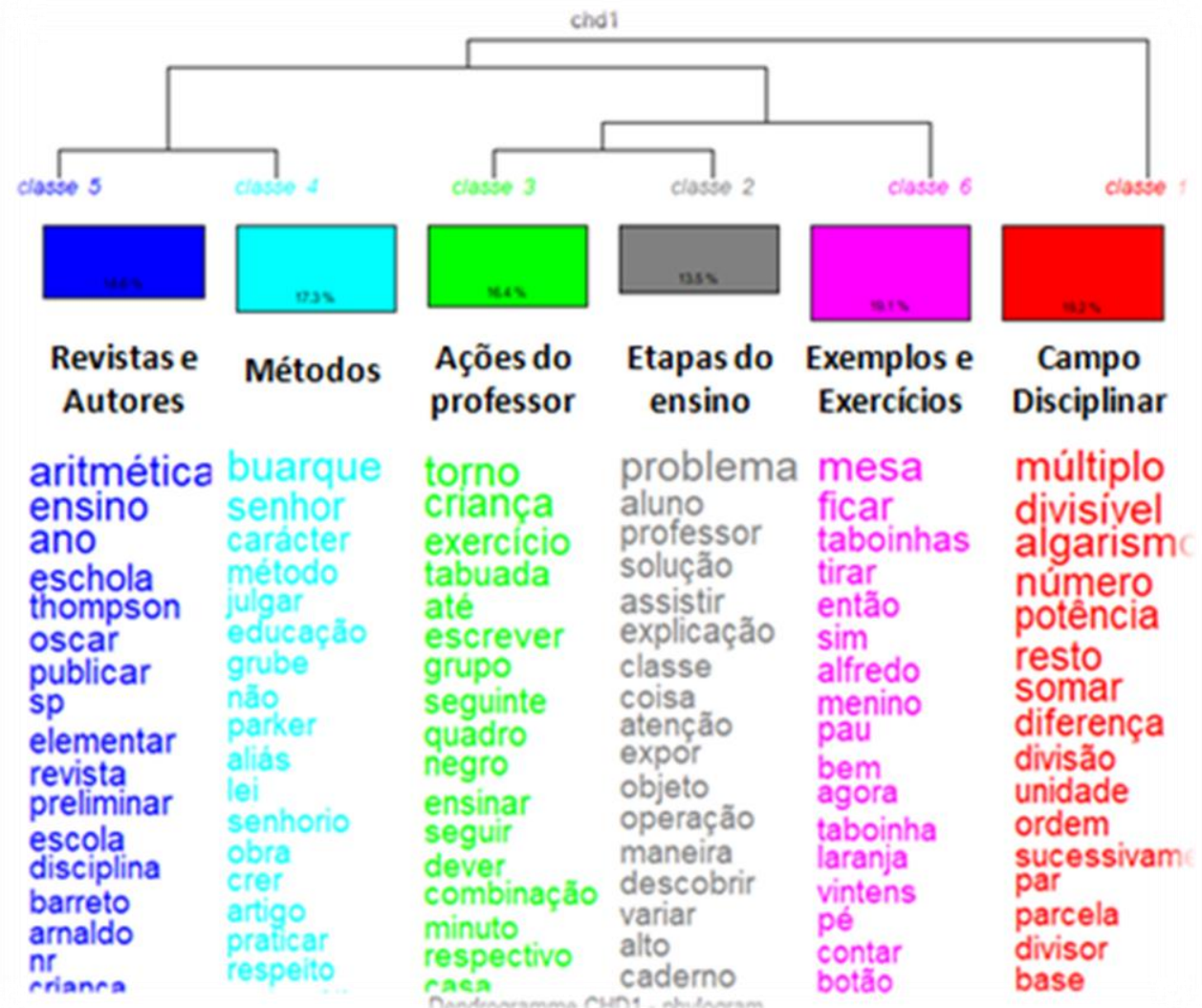

Figura 3 - CHD do corpus de aritmética gerado pelo IRAMUTEQ (as inferências do autor foram incorporadas à imagem). Fonte: IRAMUTEQ, 2019.

Quando se analisam essas quatro classes, percebe-se uma tentativa dos autores das revistas em apresentar um passo a passo a ser seguido pelo professor que ensina aritmética. Soma-se a isso a proximidade e relação de dependência entre as classes, o que evidencia uma sequência desenvolvida em conjunto entre essas classes, por exemplo, as ações do professor ao ensinar (classe 3), em diversos momentos, exige o uso de exemplos (classe 6)

Além disso, as outras duas classes, apresentaram dados para análises mais quantitativas. A classe 1 apresentou os assuntos de matemática mais abordados nos artigos, como divisão, potência, soma. Assim como a classe 5 apresentou as revistas e autores em maior evidência dentre os artigos analisados, como a revista A Eschola Publica e o autor Oscar Thompson.

É preciso enfatizar que o processo de análise para chegar ao entendimento da pesquisa ao revelar a anatomia das aulas é cíclico e não linear, os movimentos de análise passam pelo ir e vir dentro das análises estatísticas do IRAMUTEQ.

Cruzando essas inferências com pesquisas em história da educação matemática, constata-se, inicialmente, o que Carvalho (2000, p.114) já afirmara: "o impresso pedagógico didatiza-se em uma proliferação de discursos sobre os métodos ou sobre os fundamentos da prática docente". 
A Revista do Ensino e a Revista Escolar são testemunhos da longevidade de um modo de conceber e organizar o campo normativo da Pedagogia, que guarda forte relação com a concepção da pedagogia como arte de ensinar, organizando-se como caixa de utensílios e fornecendo modelos de lições e materiais para uso do professor. (CARVALHO, 2000, p. 114).

Se de um lado, as constatações de âmbito amplo já estudadas por Carvalho (2000) se fazem presentes nos resultados apresentados nesta pesquisa, este estudo não apenas chega às mesmas conclusões que a autora, como também apresenta como se dava essa tentativa de formação dos professores via revistas pedagógicas, revelando as elaborações dos autores neste movimento de formação dos professores.

Podemos dizer que o IRAMUTEQ, por meio de um modelo de classes, revelou um modelo capaz de descrever os vocabulários e conceitos envolvidos, permitindo a estruturação e sistematização de "formas de pensar" dos autores dos artigos das revistas. 0 estudo da História, por vezes, requer o entendimento do pensamento dos personagens envolvidos, objetivados em seus textos. Essa é uma das razões que leva o software criado por Pierre Ratinaud tornar-se presente em estudos das chamadas Humanidades Digitais.

\section{Considerações finais}

O estudo tratado neste texto indicou caminhos de pesquisa que permitem conjecturar sobre uma nova perspectiva de conceber de uma aula de aritmética em tempos da vaga pedagógica intuitiva. Foi possível elaborar uma anatomia, mostrando seus elementos constituintes. A análise dos textos dos autores das revistas pedagógicas paulistas mostrouse detalhada, relativamente a estudos mais gerais vindos da História da Educação. Foi possível, de algum modo, evidenciar elementos integrantes do modo de pensar a aula pelos formadores que utilizam os periódicos de modo a fazer circularem novas propostas para o ensino.

Ao trazer um modelo de pesquisa que engloba um software utilizado em estudos de Humanidades Digitais, para a história da educação matemática, inovam-se os procedimentos de investigação para essa área. Agilidade para a leitura de múltiplos documentos, análises macros e micros, apoio de cálculos estatísticos e informações exibidas de forma gráfica são ganhos que o pesquisador deve considerar como relevantes.

À medida em que novas pesquisas forem sendo desenvolvidas por meio destes modelos, haverá possibilidade de cruzamento de dados e informações possibilitando aproximações mais e mais compreensíveis relativamente à complexidade presente na cultura escolar e os significados que ela assume no desenvolvimento das práticas pedagógicas dos professores. O cruzamento de comparativos entre resultados obtidos no seio das Humanidades Digitais avizinha-se como um novo modo de produção de conhecimento sobre o passado. Um novo modo de construir, em particular, história da educação matemática. 


\section{Referências}

GOMES, V. H. L. As revistas pedagógicas paulistas e as orientações para o ensino de aritmética nos primeiros anos escolares: um estudo quali-quantitativo - São Paulo, 18901920). Dissertação (Mestrado em Ciências). São Paulo: Programa de Pós-Graduação em Educação e Saúde na Infância e na Adolescência. UNIFESP, 2020, $56 f$.

DAMASCENO, E. A. Lexicometria, geração de descritores, construção de ontologias e ensino de línguas: implicações e perspectivas. In: Magalhães, J. S. de \& Travaglia, L. C. (Org.). Múltiplas Perspectivas em Linguísticas. 1a ed. Uberlândia: EDUFU, 2008, v. 01, p. 01-3037.

BARDIN, L. Análise de conteúdo. São Paulo: Edições 70, 2011.

CARVALHO, M. M. C. Modernidade pedagógica e modelos de formação docente. São Paulo em Perspectiva. São Paulo, v. 14, n. 1, p. 111-120, 2000. 